\title{
Hepatitis B virus stimulates G6PD expression through HBx-mediated Nrf2 activation
}

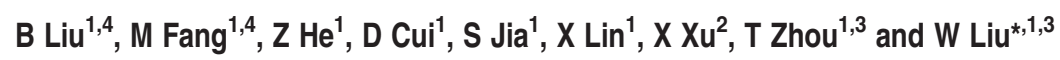

Metabolic reprogramming is a hallmark of physiological changes in cancer. Cancer cells primarily apply glycolysis for cell metabolism, which enables the cells to use glycolytic intermediates for macromolecular biosynthesis in order to meet the needs of cell proliferation. Here, we show that glucose-6-phosphate dehydrogenase (G6PD), the first and rate-limiting enzyme of the pentose phosphate pathway, is highly expressed in chronic hepatitis B virus (HBV)-infected human liver and HBV-associated liver cancer, together with an elevated activity of the transcription factor Nrf2. In hepatocytes, HBV stimulates by its X protein $(\mathrm{HBx})$ the expression of G6PD in an Nrf2 activation-dependent pathway. HBx associates with the UBA and PB1 domains of the adaptor protein p62 and augments the interaction between p62 and the Nrf2 repressor Keap1 to form HBx-p62-Keap1 complex in the cytoplasm. The aggregation of HBx-p62-Keap1 complexes hijacks Keap1 from Nrf2 leading to the activation of Nrf2 and consequently G6PD transcription. Our data suggest that HBV upregulates G6PD expression by HBx-mediated activation of Nrf2. This implies a potential effect of HBV on the reprogramming of the glucose metabolism in hepatocytes, which may be of importance in the development of HBV-associated hepatocarcinoma.

Cell Death and Disease (2015) 6, e1980; doi:10.1038/cddis.2015.322; published online 19 November 2015

Cancer is a disease with complex metabolic perturbations. Unlike normal differentiated cells that rely mainly on oxidative phosphorylation for energy production, cancer cells uptake large quantities of glucose and adopt primarily glycolysis for ATP generation even in the presence of ample oxygen. ${ }^{1}$ This metabolic characteristic promotes in cancer cells the glycolysis-associated biosynthetic processes including the pentose phosphate pathway (PPP), enabling cancer cells to utilize glucose for the biosynthesis of macromolecules to support their rapid division. ${ }^{2}$ The PPP provides cells with ribose 5-phosphate required for de novo nucleotide biosynthesis, and with the reduced form of nicotinamide adenine dinucleotide phosphate (NADPH) for reductive biosynthesis such as the production of lipid.

Glucose-6-phosphate dehydrogenase (G6PD) is the first and rate-limiting enzyme in the PPP. G6PD converts glucose6-phosphate into 6-phosphogluconolactone with a concomitant production of NADPH. Elevated G6PD expression and activity have been observed in breast, gastric, and prostatic cancers. ${ }^{3-5}$ When enhanced G6PD upregulates apoptosisinhibitory factor $\mathrm{Bcl}-2$ and $\mathrm{Bcl}-\mathrm{xl}$, and the cell cycle-related proteins, ${ }^{6}$ ectopic expression of G6PD promotes cell growth and the development of tumor in nude mice. ${ }^{7}$ It has been shown that the tumor suppressor p53 binds to G6PD and inhibits G6PD activity while many p53 mutants lost the G6PD-inhibitory activity. ${ }^{8}$ Promotion of cancer cell proliferation by TAp73, a p53-related protein, is also attributed to an upregulated G6PD. ${ }^{9}$ These data suggest that in addition to contribute to cancer growth and survival, G6PD also serves as an oncogene.

NF-E2-related factor 2 (Nrf2) is a master transcriptional factor responsible for the regulation of a number of antioxidant and cytoprotective genes, primarily in response to electrophiles and reactive oxygen species (ROS). ${ }^{10}$ Under normal conditions, Nrf2 is constantly associated with its inhibitor Kelch-like $\mathrm{ECH}$-associated protein 1 (Keap1) and degraded by the proteasomes. Elevated intracellular ROS and accumulation in electrophiles lead to oxidation of key cysteine residues on Keap1 disrupting Keap1-Nrf2 interaction. Nrf2 then shifts into the nucleus and activates the transcription of cytoprotective genes that encode detoxifying enzymes. Recently, accumulating evidence has demonstrated a constitutive stabilization of Nrf2 in various human cancers; ${ }^{11-14}$ and cancers with high Nrf2 level are associated with poor prognosis. ${ }^{10,11}$ In addition, elevated Nrf2 activity enhances the expression of PPP enzymes including G6PD, and accelerates cancer cell proliferation. ${ }^{15}$ Deletion of Nrf2 can reduce carcinogen-induced lung tumor development in mice ${ }^{16}$ and the oncogenes $K$-Ras and Myc specifically target the expression of Nrf2 in cancer cells. ${ }^{17}$ These data suggest that Nrf2 is an important mediator of oncogenesis. Intriguingly, it has recently been shown that accumulation of p62, an

\footnotetext{
${ }^{1}$ Department of Biochemistry and Molecular Biology, Program in Molecular Cell Biology, Zhejiang University School of Medicine, Hangzhou, Zhejiang 310058, China; ${ }^{2}$ Department of Surgery, First Affiliated Hospital, Zhejiang University School of Medicine, Hangzhou, Zhejiang 310003, China and ${ }^{3}$ Collaborative Innovation Center for Diagnosis and Treatment of Infectious Disease, First Affiliated Hospital, Zhejiang University School of Medicine, Hangzhou, Zhejiang 310003, China

*Corresponding author: W Liu, Department of Biochemistry and Molecular Biology, Zhejiang University School of Medicine, Building B, Room 720, 866 Yu-Hang-Tang Road, Hangzhou, Zhejiang 310058, China. Tel/Fax: +86 571 88208357; E-mail: liuwei666@zju.edu.cn

${ }^{4}$ These authors contributed equally to this work.

Abbreviations: HBV, hepatitis B virus; HCC, hepatocellular carcinoma; HBx, hepatitis B virus X protein; G6PD, glucose-6-phosphate dehydrogenase; Nrf2, NF-E2-related factor 2; ROS, reactive oxygen species; Keap1, kelch-like ECH-associated protein 1; PPP, pentose phosphate pathway; HBcAg, hepatitis B core antigen; qRT-PCR, quantitative reverse transcription PCR; FRAP, fluorescence recovery after photobleaching; FLIP, fluorescence loss in photobleaching; Nq01, NAD(P)H quinone oxidoreductase 1; Gstm1, glutathione S-transferase M1; Cyp2a5, cytochrome P450 2A5; PB1, Phox and Bem1p; UBA, ubiquitin-associated; ZZ, ZZ-type zinc finger Received 27.5.15; revised 19.8.15; accepted 10.9.15; Edited by H-U Simon
} 
autophagy-adaptor protein, can cause a persistent activation of Nrf2 contributing to the growth of human hepatocellular carcinoma $(\mathrm{HCC}) .^{14,18}$

In this study, using clinical specimen and cultured cells, we have investigated the potential influence of hepatitis $B$ virus (HBV), a major pathogenic factor for HCC worldwide, on the metabolism of hepatocytes by focusing on the expression of G6PD. We found that HBV upregulates G6PD in hepatocytes which relies on its $X$ protein $(\mathrm{HBX})$-mediated activation of Nif2. HBx interacts with p62 and Keap1 to generate $\mathrm{HBx}-\mathrm{p} 62-\mathrm{Keap} 1$ aggregates in the cytoplasm leading to the nuclear translocation and activation of Nrf2.

\section{Results}

HBV upregulates G6PD expression. To test whether HBV infection may potentially modulate the PPP in hepatocellular metabolism, we began with the investigation of the expression of G6PD, the rate-limiting enzyme of the PPP, in liver tumor and non-tumor tissues from subjects with HBV-associated HCC, and in samples from normal control individuals. We found that the G6PD mRNA level in the tumor $(10 / 13=76.9 \%)$ and non-tumor tissues $(7 / 13=53.8 \%)$ dramatically increased compared with the normal group (Figure 1a and Supplementary Figure 1a). Immunostaining
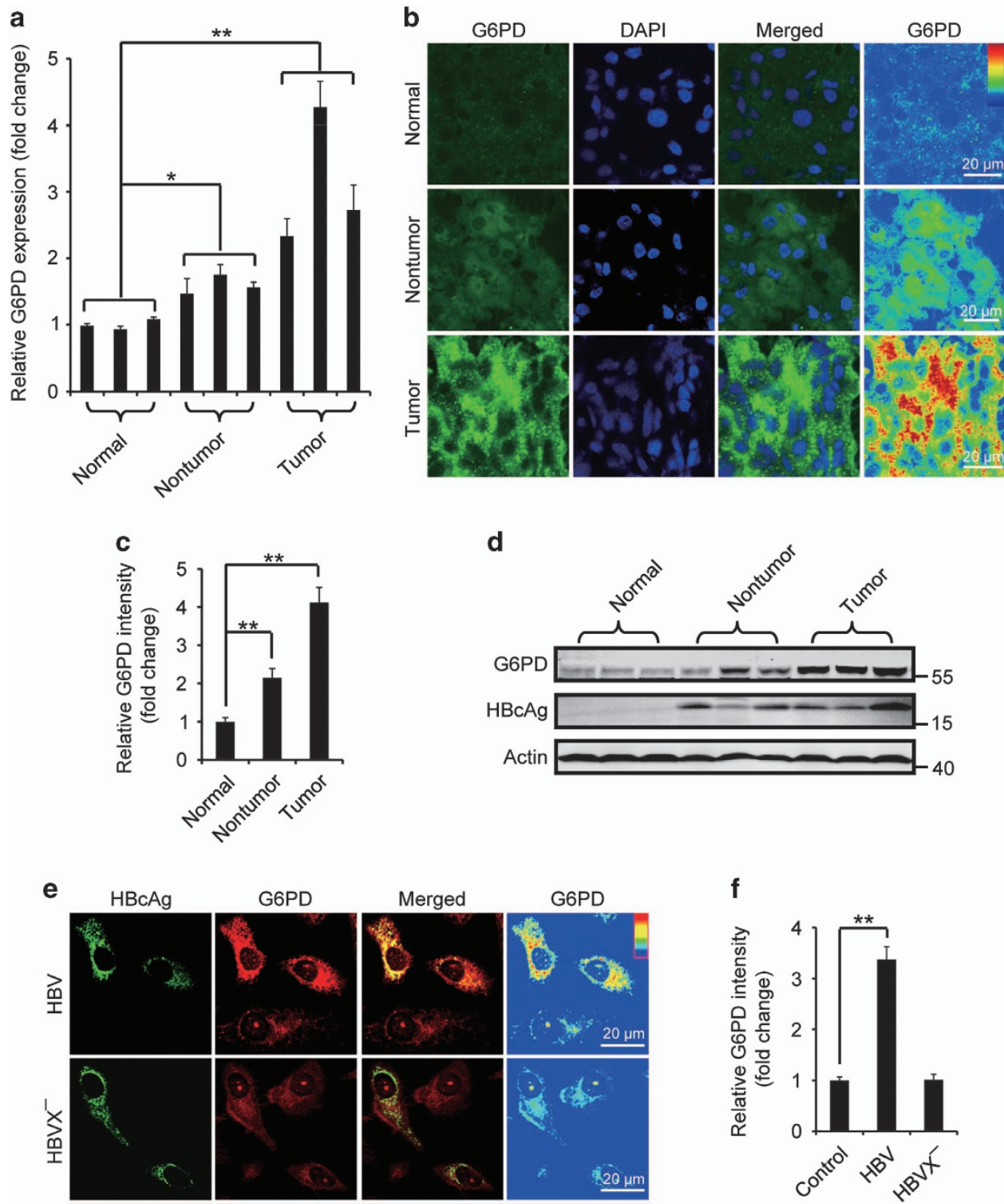

Figure 1 HBV upregulates G6PD expression through HBx protein. (a) The relative G6PD mRNA levels in normal liver tissues, liver tumor or non-tumor tissues. The data are presented as mean \pm S.E.M. of triplicate experiments. (b) Representative immunostaining of G6PD in the human tissues. (c) Quantification of the relative mean G6PD intensity from 10 randomly selected visual fields in each human tissue. (d) Western blot analysis of G6PD in the human tissues. (e) Huh7 cells were transfected with the HBV genomic DNA $(\mathrm{HBV})$ or the $\mathrm{HBX}$-negative $\mathrm{HBV}$ genomic DNA $\left(\mathrm{HBVX}^{-}\right)$. At $48 \mathrm{~h}$ after transfection, the cells were stained with $\mathrm{HBcAg}$ and $\mathrm{G} 6 \mathrm{PD}$ antibodies. (f) Quantification represents the relative fluorescence intensity of G6PD in cells with or without expression of $\mathrm{HBV}$ or $\mathrm{HBVX}^{-}, n=30$. All the quantitative data are presented as mean $\pm \mathrm{S}$.E.M., ${ }^{* *} P<0.01$, ${ }^{*} P<0.05$. Scale bars, $20 \mu \mathrm{m}$ 
and western blot affirmed an elevation of G6PD protein level in the same samples while the tumor samples showed a higher G6PD than that in the non-tumor samples (Figures $1 b-d)$. In search of evidence that HBV infection stimulates G6PD expression, we checked G6PD protein in human hepatoma Huh7 cells expressing HBV genomic DNA, and HepG2.2.15 cell, a HBV-replicating cell line. We found in the cells that HBV DNA expression dramatically raised G6PD protein level (Figures 1e and $f$ and Supplementary Figure 1b). Interestingly, expression of $\mathrm{HBVX}^{-}$DNA, an HBV genomic DNA that is incapable of expressing $\mathrm{HBx}$ protein, failed to increase G6PD level, suggesting a critical role of $\mathrm{HBx}$ in the stimulation (Figures 1e and f).

To verify the role of $\mathrm{HBx}$ in mediating HBV's effect on G6PD expression, hepatitis $B$ surface antigen ( $\mathrm{HBsAg})$, hepatitis $B$ core antigen $(\mathrm{HBcAg})$ or $\mathrm{HBx}$, was transfected respectively in human hepatic L02 cells and Huh7 cells. Significantly, HBx, but not HBsAg or HBcAg, hiked G6PD at both mRNA and protein levels in L02 and Huh7 cells (Figures $2 a$ and $b$ and Supplementary Figures $1 \mathrm{c}$ and $\mathrm{d}$ ). Considering the higher transfection efficiency, Huh7 cells were used in subsequent investigation. Using glucose-6-phosphate as a substrate, enzyme activity analysis demonstrated a boosted total G6PD activity in HBx-Myc-transfected cells (Figure 2c). Taken together, these results suggest that HBV upregulates G6PD in hepatocytes through HBx.
HBV stimulates Nrf2 activation. Nrf2 contributes to cancer development by targeting not only the typical cytoprotective genes but also genes involved in cell metabolism including G6PD. ${ }^{15}$ To test a potential role of Nrf2 in HBV-triggered upregulation of G6PD, we performed Nrf2 RNA interference and checked G6PD expression and activity in Huh7 cells. Knockdown of Nrf2 evidently decreased the mRNA and protein levels and total activities of G6PD induced by $\mathrm{HBx}-\mathrm{Myc}$ expression (Figures 2d-f), indicating wherein an essential role of Nrf2. We then investigated the effect of $\mathrm{HBV} / \mathrm{HBx}$ on Nrf2 activity. First, we looked over the intracellular distribution of Nrf2. In control HepG2 cells, Nrf2 is primarily localized to the cytoplasm. But in HepG2.2.15 cells Nrf2 showed a typical nuclear distribution (Figure 3a), indicating an activation of Nrf2. We also measured the expression of known Nrf2 target genes including $\mathrm{NAD}(\mathrm{P}) \mathrm{H}$ quinone oxidoreductase 1 (Nqo1), glutathione S-transferase M1 (Gstm1), and cytochrome P450 2A5 (Cyp2a5) in HepG2 and HepG2.2.15 cells. Apparently, HepG2.2.15 cells possessed higher level of each of the genes compared with that in HepG2 cells (Figure $3 b$ ). Finally, we transfected in the cells the HBV DNA and HBVX- DNA and checked Nrf2 distribution. When Nrf2 showed a typical cytoplasmic distribution in non-transfected cells or cells expressing $\mathrm{HBVX}^{-}$DNA, Nrf2 in HBV DNA-expressing cells was localized primarily to the nucleus (Figure $3 \mathrm{c}$ ), indicating an HBx-mediated Nrf2 activation. This action of $\mathrm{HBx}$ was a

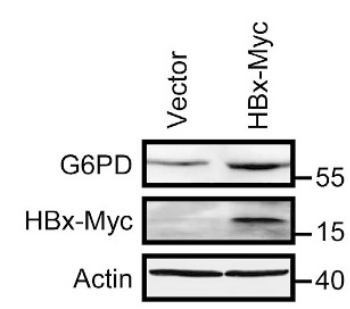

d

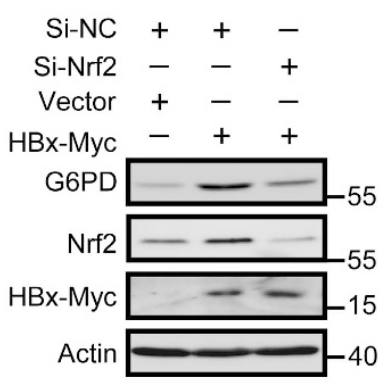

b

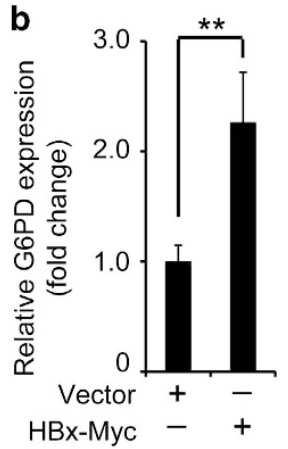

e

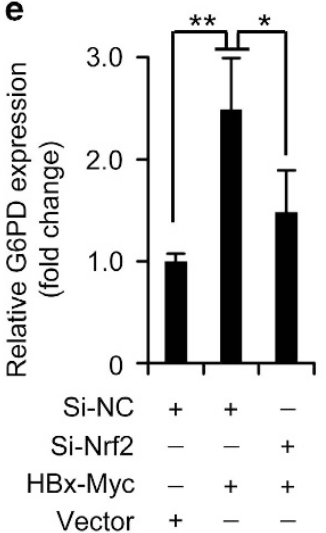

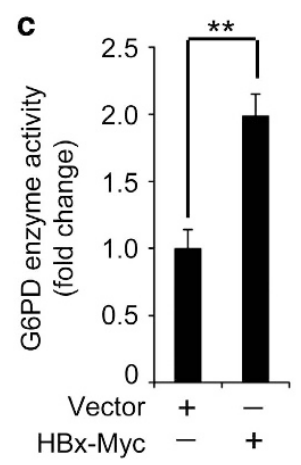

f

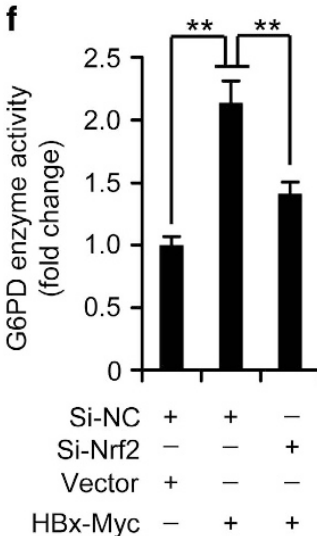

Figure 2 HBx enhances G6PD activity via Nrf2. (a and $\mathbf{b}$ ) Western blot (a) and qRT-PCR (b) analysis of G6PD expression in Huh7 cells expressing HBx-Myc. (c) The relative G6PD enzyme activity in cells treated as in (a). (d-f) The protein level (d), mRNA level (e), and enzyme activity (f) of G6PD in Huh7 cell with or without HBx-Myc expression and Nrf2 RNAi. All the statistical data are presented as mean \pm S.E.M. of triplicate experiments. ${ }^{*} P<0.05 ;{ }^{\star} P<0.01$ 
a
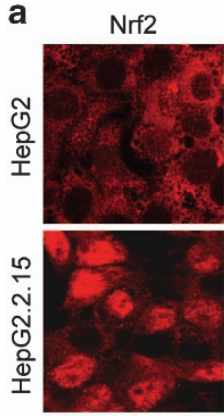

c
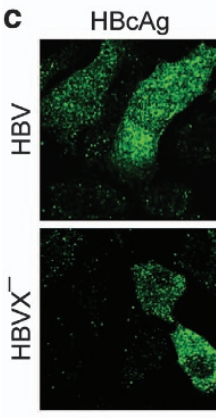

d

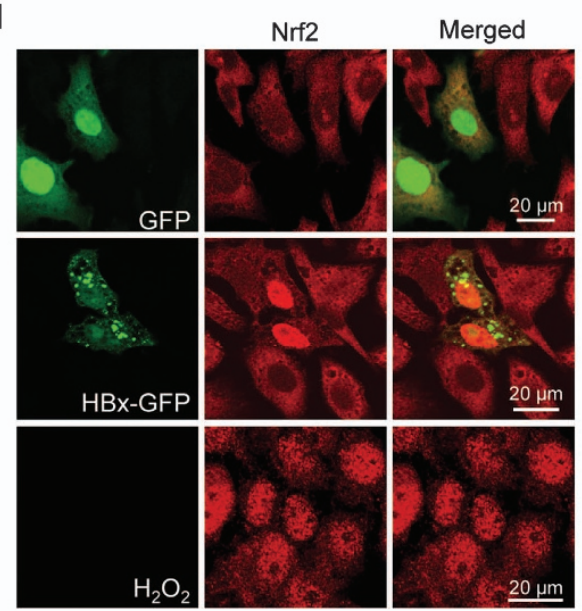

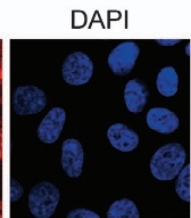
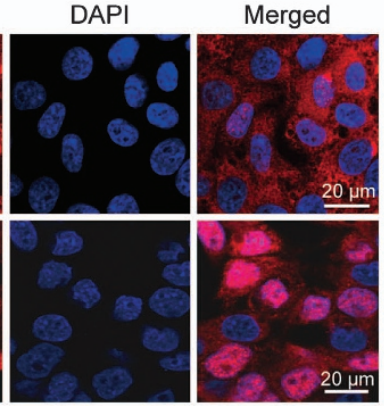
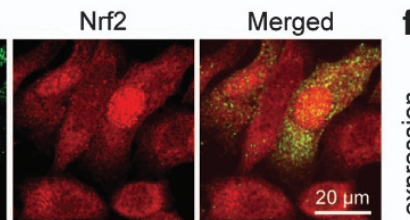

b

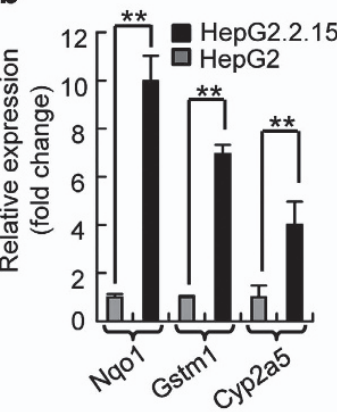

e

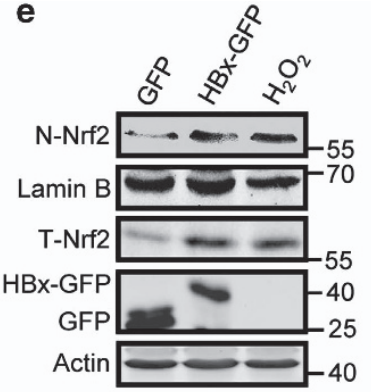

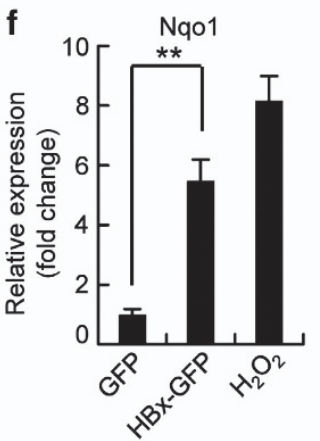
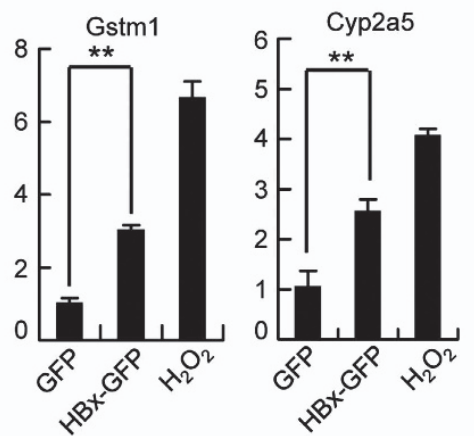

g
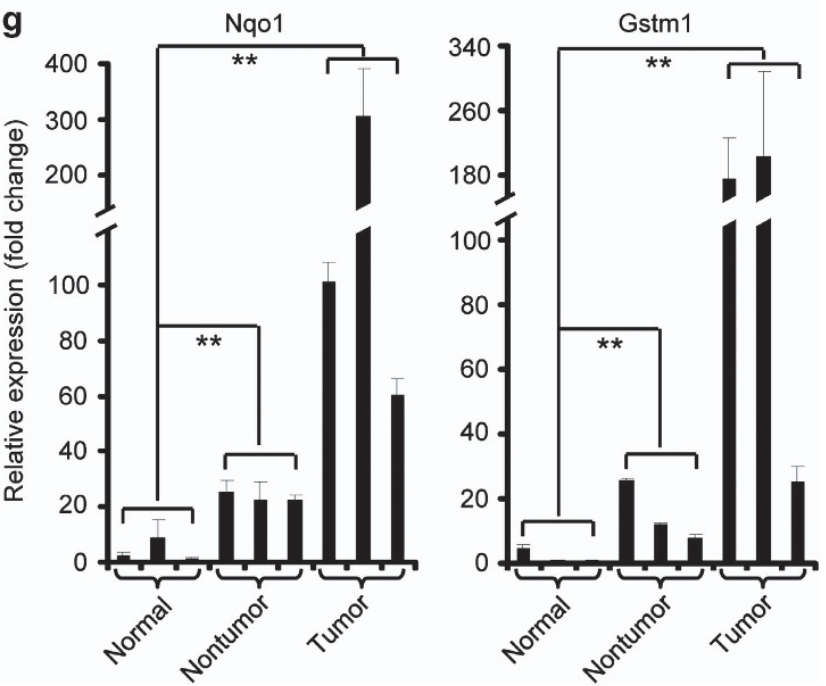

Figure 3 HBV stimulates Nrf2 activation. (a) Immunostaining of Nrf2 in HepG2 and HepG2.2.15 cells. Note the nuclear distribution of Nrf2 in HepG2.2.15 cells. (b) The mRNA levels of Nqo1, GSTm1, and Cyp2a5 measured by qRT-PCR in HepG2 and HepG2.2.15 cells. (c) Huh7 cells transfected with HBV genomic DNA (HBV) or HBx-negative HBV

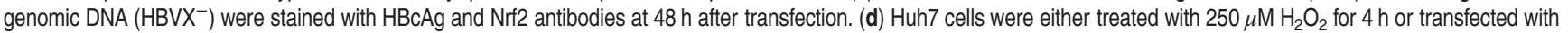
GFP or HBx-GFP for $48 \mathrm{~h}$. Then, the cells were fixed and stained with Nrf2 antibody. (e) Western blot analysis of Nrf2 protein in nuclear extracts (N-Nrf2) and cell lysis (T-Nrf2) in cells treated as in (d). Lamin B was used as an internal control of nuclear fraction. (f) The mRNA levels of Nqo1, GSTm1, and Cyp2a5 measured by qRT-PCR in cells treated as in (d). (g) Nqo1 and GSTm1 mRNA levels in normal liver tissues and HBV-infected non-tumor or tumor tissues. All the statistical data are presented as mean \pm S.E.M. of triplicate experiments. ${ }^{*} P<0.01$. Scale bars, $20 \mu \mathrm{m}$

confirmed in cells expressing GFP-tagged HBx (HBx-GFP). Similar to that in cells treated by $\mathrm{H}_{2} \mathrm{O}_{2}$, a classic Nrf2 activator, expression of $\mathrm{HBx}$, but not $\mathrm{HBsAg}$ or $\mathrm{HBcAg}$, shifted cytoplasmic Nrf2 to the nucleus in L02 and Huh7 cells (Figure $3 \mathrm{~d}$ and Supplementary Figure 2). The nuclear translocation of Nrf2 was further proved by a subcellular fractionation showing increased Nrf2 in the nucleus of $\mathrm{HBx}$ - expressing Huh7 cells (Figure 3e). Consistently, the expression of Nrf2 target genes, Nqo1, Gstm1, and Cyp2a5, was enhanced by HBx-GFP expression (Figure 3f).

Nrf2 activity was also examined in liver tissues from subjects with or without HBV-associated HCC by measuring the expression of Nrf2 target genes. Compared with the normal samples from healthy individuals, the non-tumor and 
tumor samples from HCC patients showed increased expression of the genes representing augmented Nrf2 activity (Figure 3g).

Taken together, these results suggest that HBV stimulates Nrf2 activation in hepatocytes.

HBx stimulates p62-Keap1 interaction. Because the Nrf2 activity is mainly regulated by its interaction with Keap1, to investigate the mechanism by which $\mathrm{HBx}$ stimulates Nrf2 activation, we began with the observation of intracellular Keap1 localization. In Huh7 cells, expression of HBx-GFP but not GFP as a control, when endogenous cytoplasmic Nrf2 shifted to the nucleus, in the same cells, coexpressed Dsred-Keap1 formed in the cytoplasm aggregates with HBx-GFP (Figure 4a), implying an interaction between Keap1 and HBx. We then tested the potential role of p62 in mediating the Keap1-HBx interaction, because $\mathrm{HBx}$ can cause in hepatocytes the accumulation of $\mathrm{p} 62,{ }^{19}$ and elevated p62 results in Keap1 aggregation and Nrf2 activation through interaction with Keap1.20 We found a colocalization of p62 to Keap1-HBx aggregates in HBx-GFP and Dsred-Keap1-coexpressing cells (Figure 4b). In addition, in HBx-expressing cells, co-immunoprecipitation of p62 and Keap1 dramatically increased, indicating an enhanced interaction of the two proteins (Figure 4c). Promoted p62-Keap1 interaction was also observed in HepG2.2.15 cells compared with HepG2 cell (Supplementary Figure 3a). Furthermore, in liver tissue samples, in contrast to the normal liver tissue in which Keap1 and p62 were dispersed in the cytoplasm, the tumor and non-tumor samples from HBV-associated HCC patients showed a palpable colocalization of p62 and Keap1 forming in the cytoplasmic the p62-Keap1 aggregates, with more such aggregates in the tumor tissues (Figure $4 d$ ). These data suggested a stimulating effect of HBV on p62-Keap1 interaction through HBx. We further examined the $\mathrm{HBx}$-Keap1 interaction in p62-deficient cells. In Huh7 cells expressing HBx-GFP, immunoprecipitation of HBx using GFP antibody resulted in a co-precipitation of endogenous Keap1 and p62 (Figure 4e). However, HBx-GFP failed to co-precipitate Keap1 in p62 knockdown cells or in cells overexpressing p62T352A, a p62 mutant defective in Keap1 interaction, ${ }^{20}$ while a strong co-precipitation of p62T352A with $\mathrm{HBx}$ was still detectable (Figure 4e). In addition, in p62 knockdown cells or cells expressing Flag-p62T352A, Keap1 could no more be recruited to the HBx punctual aggregates (Figure 4f). These data suggest that $\mathrm{HBx}$ can drive in hepatocytes the formation of HBx-p62-Keap1 complexes in which the recruitment of Keap1 relies on its interaction with p62.

At last, we examined the role of $\mathrm{HBx}-\mathrm{p} 62-$ Keap1 complex formation in the $\mathrm{HBV} / \mathrm{HBx}$-stimulated Nrf2 activation, by knocking down p62 in HepG2.2.15 cells and HBx-expressing Huh7 cells. Significantly, knockdown of p62 decreased Nqo1 expression in both cells (Figure $4 \mathrm{~g}$ and Supplementary Figure 3b). The activity of G6PD was also attenuated by 062 RNAi in Huh7 cells expressing GFP-HBx (Figure 4h). These results therefore suggest a crucial role of the formation of $\mathrm{HBx}$-p62-Keap1 complexes in HBx-triggered Nrf2 activation and G6PD expression.
Immobilization of Keap1 in the HBx-p62-Keap1 aggregates. Given that $\mathrm{HBx}$ interacts with p62 and Keap1 to form HBx-p62-Keap1 aggregates in the cytoplasm, and the interactions contribute to the HBV-stimulated Nrf2 activation, a possible mechanism is that Keap1 is stuck in the aggregates leading to an abated inhibition of Nrf2. To address this, we checked whether Keap1 is associated with the aggregates in a dynamic on/off or in/out manner. First, we carried out fluorescence recovery after photobleaching (FRAP) in living cells expressing GFP-Keap1. A cytoplasmic region of GFP-Keap1 was photobleached and recovery of the fluorescence was monitored over time. Upon photobleaching, we observed a rapid recovery from the non-bleached pool, with the original prebleached fraction of fluorescence clearly restored in less than $100 \mathrm{~s}$ (Figures $5 \mathrm{a}$ and e). In contrast, in GFP-Keap1 and HBx-Cherry cotransfected cells, the fluorescence recovery of GFP-Keap1 aggregated with HBx-Cherry was dramatically slowed, with a great proportion of the initial GFP-Keap1 fluorescence failing to recover, even in a much longer time after photobleaching (Figures $5 b$ and e), suggesting that this proportion of Keap1 was irreversibly bound to the aggregates. The irreversible binding of Keap1 to the aggregated pool was further confirmed by fluorescence loss in photobleaching (FLIP). GFP-Keap1 fluorescence in a small cytoplasmic region of the cell expressing GFP-Keap1 and Cherry was bleached repetitively. After about $200 \mathrm{~s}$, the fluorescence signals were completely lost in the areas outside the region, indicating rapid diffusion between the bleached and unbleached areas (Figures $5 \mathrm{c}$ and f). However, in cells expressing GFP-Keap1 and HBx-Cherry, repetitive photobleaching of a similar cytoplasmic pool failed to cause the loss of GFP-Keap1 from the GFP-Keap1 and HBx-Cherry puncta, indicating a fixation of GFP-Keap1 in the aggregates (Figures $5 \mathrm{~d}$ and $\mathrm{f}$ ). These results suggest that the HBx-p62Keap1 complexes are able to immobilize Keap1 in the formed aggregates.

To further investigate whether fix of Keap1 in the HBx-p62Keap1 aggregates contributes to HBx-triggered Nrf2 activation, we created different truncated p62 mutants (Figure $5 \mathrm{~g}$ ), and the HBx-p62-Keap1 aggregate formation and Nrf2 activation were analyzed. We found that when coexpressed in cells, HBx and p62 $\Delta Z Z$, a mutant p62 lacking the ZZ-type zinc finger domain, and Keap1, can still interact with each other to form the aggregates (Figure $5 \mathrm{~h}$ and Supplementary Figure 4). However, in cells coexpressing HBx with p62 $\triangle U B A$ or p62 $\triangle \mathrm{PB} 1$, two $\mathrm{p} 62$ mutants lacking the ubiquitin-associated domain or the Phox and Bem1p domain that fail to aggregate, ${ }^{21} \mathrm{HBx}$ was not able to interact with Keap1 (Supplementary Figure 4), and they failed to form in the cytoplasm the aggregates (Figure 5h). Correspondingly, expression of p62 $\mathrm{U}$ BA or p62 $\triangle \mathrm{PB1}$, but not p62 $\triangle \mathrm{ZZ}$, disrupted $\mathrm{HBx}$-stimulated expression of Nrf2-targeted genes Nqo1 and Cyp2a5 (Figure 5i). These data suggest that by immobilization of Keap1, the formation of HBx-p62-Keap1 complex initiated by $\mathrm{HBx}$ is essential to $\mathrm{HBx}$-stimulated Nrf2 activation.

Nrf2 and G6PD are required for HBx-promoted cell proliferation. Both Nrf2 and G6PD promote tumorigenesis. ${ }^{7,14}$ To investigate a potential involvement of $\mathrm{HBx}$-stimulated Nrf2 
activation and G6PD expression in HBV-associated hepatocarcinogenesis, we checked the proliferation of HepG2 cells stably expressing $\mathrm{HBx}-\mathrm{GFP}$, with or without Nrf2 or
G6PD knockdown. Consistent with previous reports, ${ }^{22-24}$ HBx-GFP expression enhanced proliferation of HepG2 cells (Figure 6b), and knockdown of Nrf2 or G6PD

a

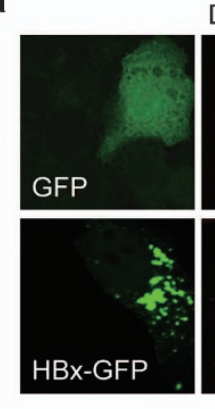

Dsred-Keap1
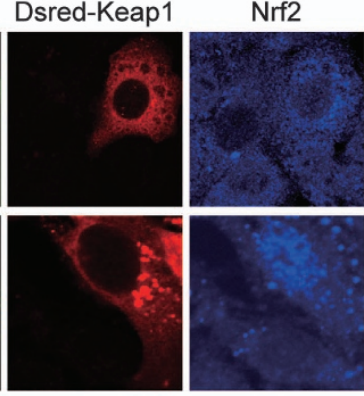

b

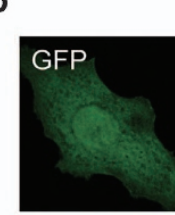

Dsred-Keap1
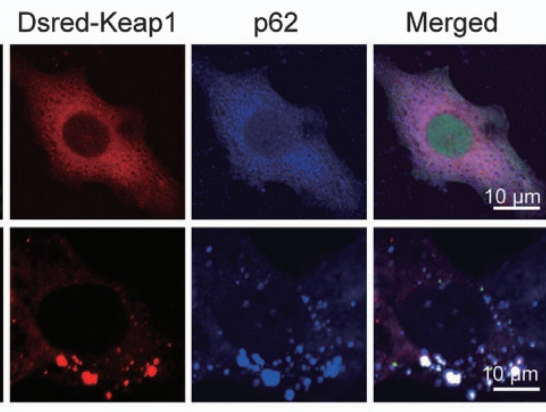

C
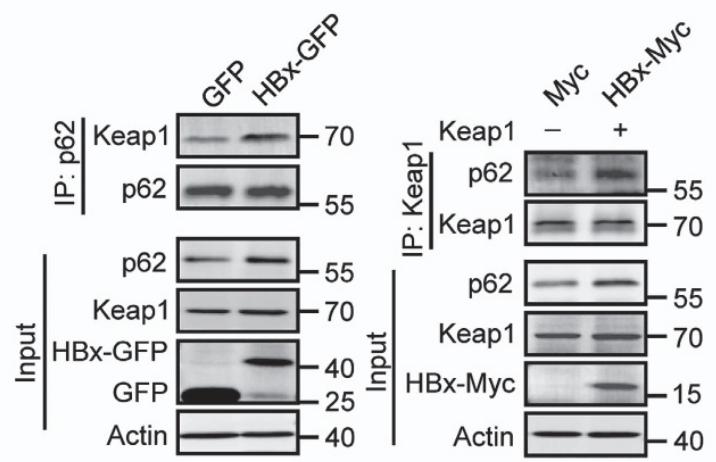

f
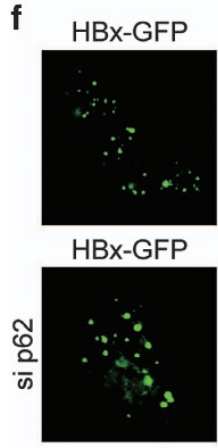

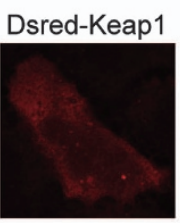

Dsred-Keap1

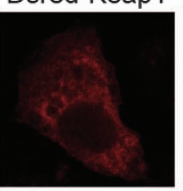

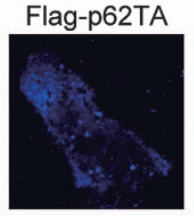

p62

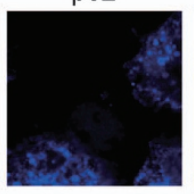

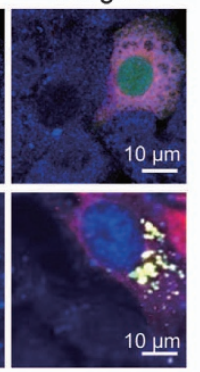

$\underline{\mu m}$
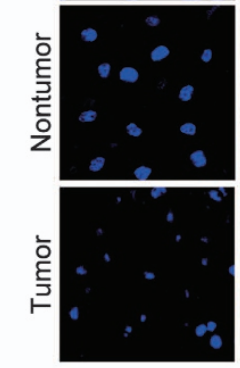

d

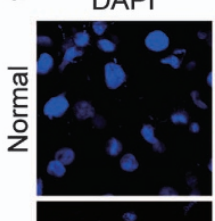

Keap1
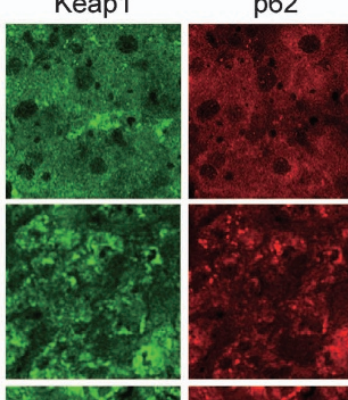

Merged

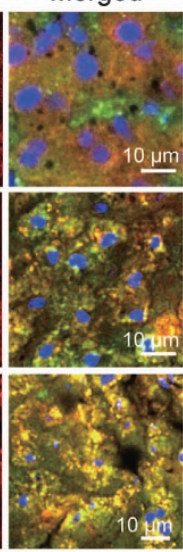

Figure 4 HBx-stimulated p62-Keap1 interaction is required for HBx-induced Nrf2 activation. (a) Localization of Dsred-Keap1 and Nrf2 in HBx-GFP-transfected Huh7 cells. (b) Colocalization of Dsred-Keap1 and p62 in HBx-GFP-expressing Huh7 cells. (c) Co-immunoprecipitation of Keap1 with p62 (left panel) or p62 with Keap1 (right panel) in HBxexpressing cells. (d) Immunostaining of p62 and Keap1 in normal liver tissues and HBV-infected non-tumor or tumor tissues. (e) Co-immunoprecipitation of HBx-GFP, p62, and Keap1 in HBx-GFP-expressing cells transfected without or with p62 siRNAs, Flag-p62, or Flag-p62T352A (p62TA). (f) Localization of Dsred-Keap1 in HBx-GFP-transfected cells with Flag-p62TA expression (upper panel) or p62 RNAi (lower panel). (g) Nq01 mRNA level measured by qRT-PCR in HBx-GFP-expressing cells with or without p62 RNAi. (h) G6PD enzyme activity in cells treated as in (g). All the statistical data are presented as mean \pm S.E.M. of triplicate experiments. ${ }^{\star \star} P<0.01$. Scale bars, $10 \mu \mathrm{m}$ 
a
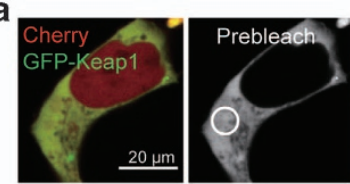

b

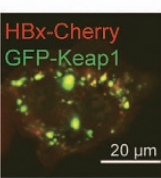

C

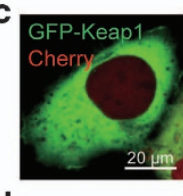

d

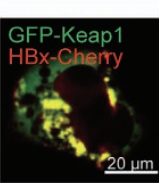

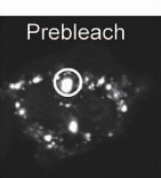
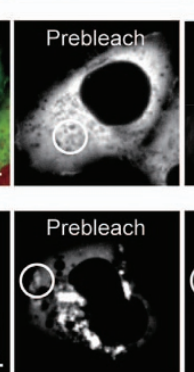
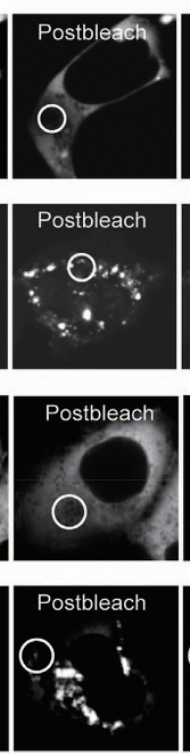
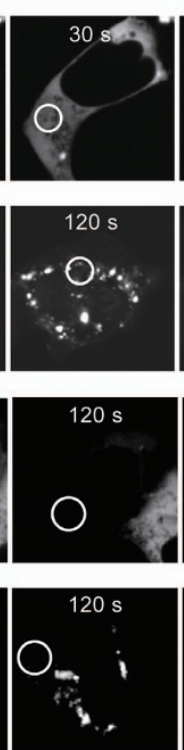
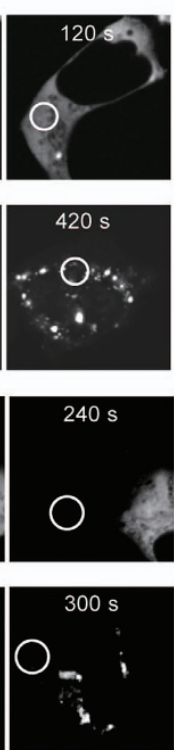
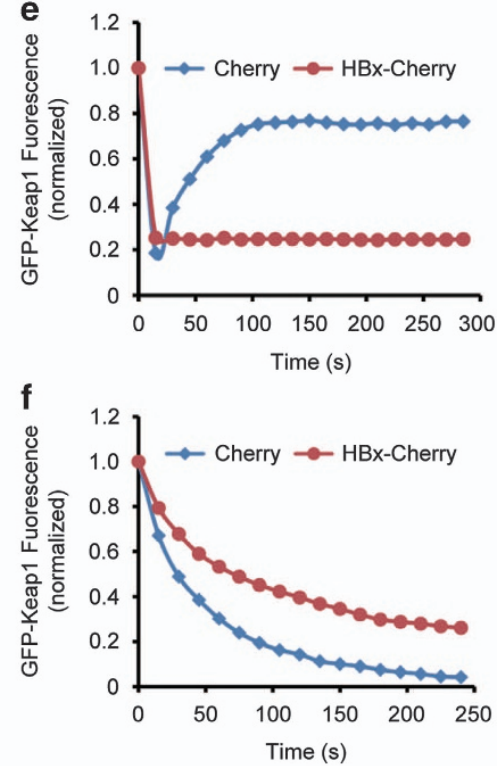

g

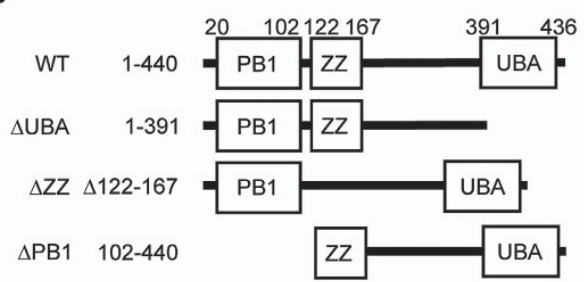

i

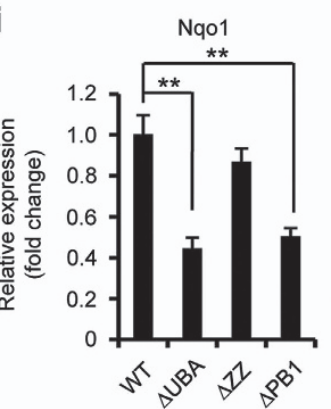

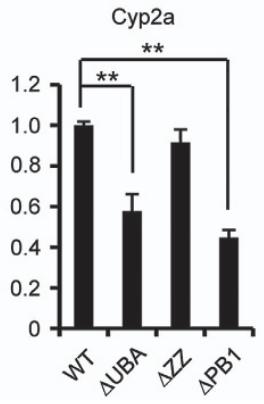

h
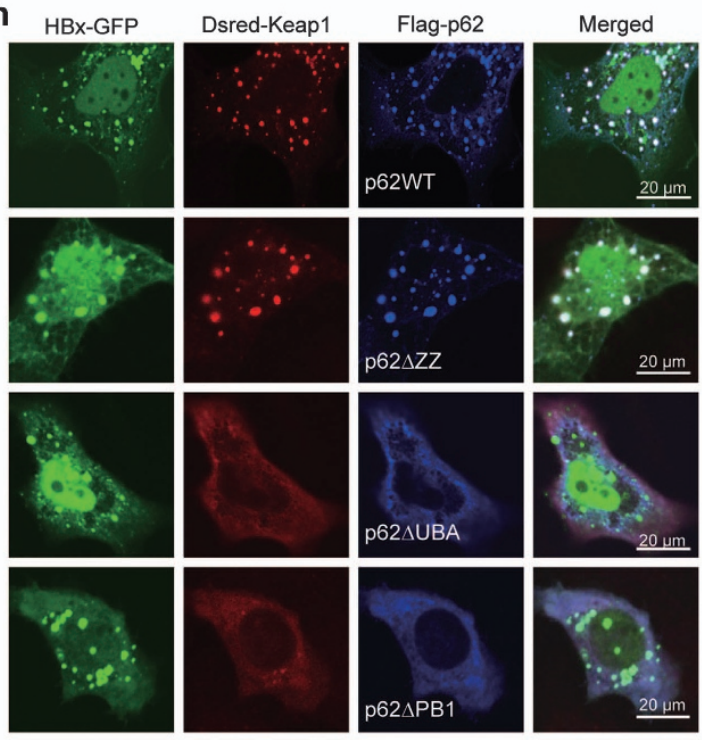

Figure 5 HBx immobilizes Keap1 in HBx-p62-Keap1 aggregates. (a and b) Huh7 cells expressing Cherry and GFP-Keap1 (a) or HBx-Cherry and GFP-Keap1 (b) were imaged before and after photobleaching the indicated region (white circles) by high intensity $488 \mathrm{~nm}$ laser light. Note the speed of fluorescence recovery in the photobleached region. (c and d) Repeated photobleaching of a cytoplasmic region (white circles) led all GFP-Keap1 fluorescence to be lost within a cell expressing GFP-Keap1 and Cherry (c), but not from the GFP-Keap1-HBx-Cherry aggregates in a cell expressing GFP-Keap1 and HBx-Cherry (d). (e) Quantification of the GFP-Keap1 signals in (a) and (b). (f) Quantification of the GFP-Keap1 signals in (c) and (d). (g) Schematic representation of the structure of p62 and the truncated mutants. (h) Localization of Dsred-Keap1 in cells expressing HBx-GFP with Flag-p62 or each of the Flag-tagged truncated p62 mutants. (i) Relative mRNA levels of Nq01 and Cyp2a5 measured by qRT-PCR in cells transfected with $\mathrm{HBx}$ and $\mathrm{p} 62$ or $\mathrm{HBx}$ and each of the truncated p62 mutants. Data are presented as mean \pm S.E.M. of triplicate experiments. ${ }^{*} P<0.01$. Scale bars, $20 \mu \mathrm{m}$

significantly inhibited HBx-GFP-stimulated cell proliferation (Figures $6 a$ and b). We also performed colony formation assay to dissect the function of Nrf2 and G6PD. We found that knockdown of either Nrf2 or G6PD significantly repressed $\mathrm{HBx}$-stimulated colony formation of HepG2 cells in soft agar (Figure 6c and Supplementary Figure 5).

\section{Discussion}

Chronic HBV infection is worldwide one of the major causes for primary liver cancer, and the molecular mechanism for
HBV-associated HCC remains elusive. Here, we demonstrate that HBV enhances in hepatocytes the expression of G6PD, the rate-limiting enzyme of the PPP, by activating Nrf2. Formation of $\mathrm{HBx}-\mathrm{p} 62-\mathrm{Keap} 1$ aggregates in the cells that hijack Keap1 from Nrf2 underlies the mechanisms of HBV-stimulated Nrf2 activation for G6PD expression.

$\mathrm{HBx}$ has been suggested as a major pathogenic protein for HBV-associated HCC. However, previous studies have mostly focused on the action of $\mathrm{HBx}$ in the intracellular signal transduction pathways. HBx-mediated upregulation of G6PD and potential reprogramming of hepatocyte metabolism may 
a

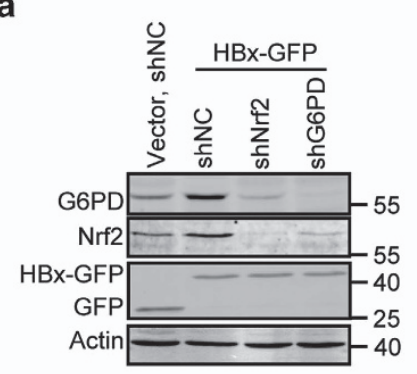

b

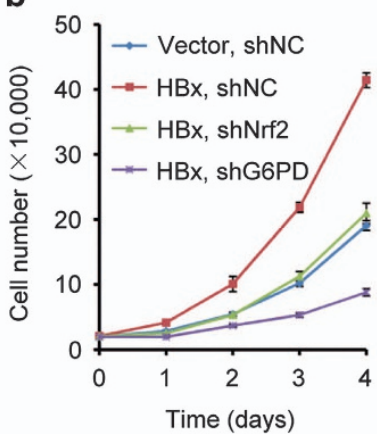

C

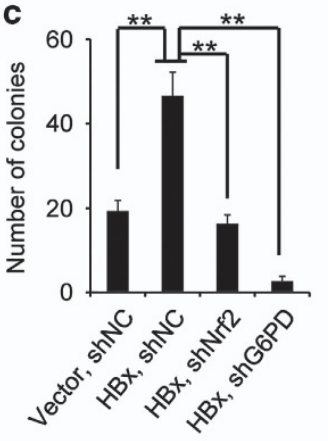

d Normal condition

HBV infection

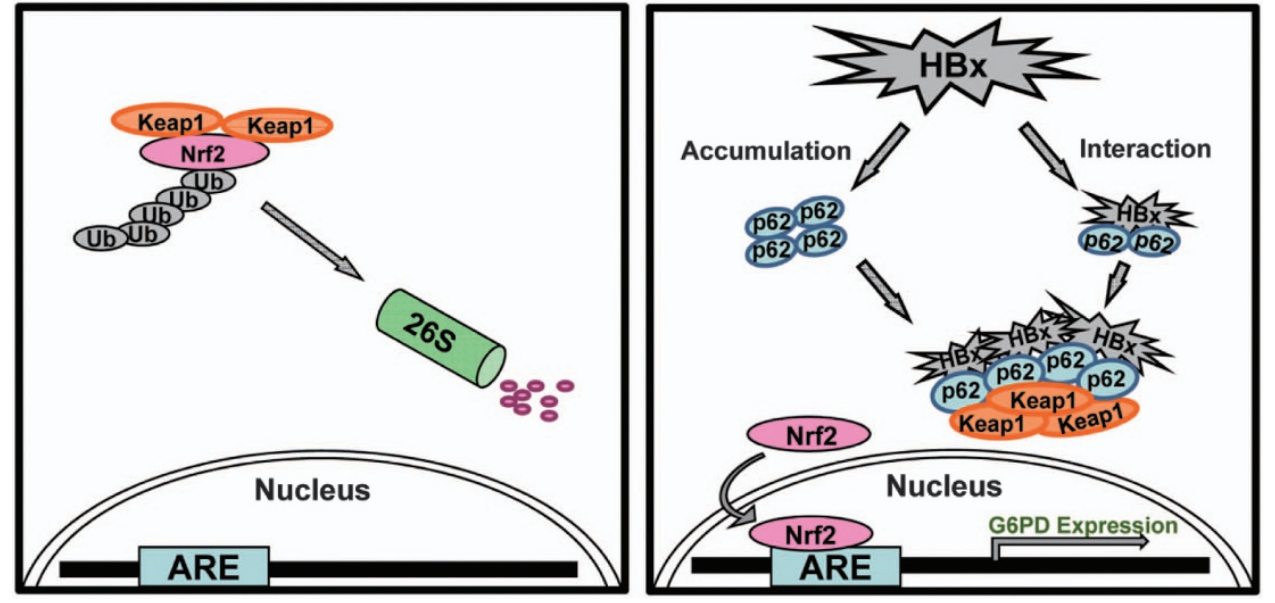

Figure 6 Nrf2 and G6PD are required to HBx-promoted cell proliferation. (a) G6PD and Nrf2 protein levels in HBx-GFP cells with the expression of indicated shRNAs. (b) Cell proliferation assay of the cells in (a). Data are presented as mean \pm S.E.M. of triplicate experiments. (c) Colony formation assay of the cells in (a). Colonies in three randomly chosen fields per well were quantified. Data are presented as mean \pm S.E.M. of triplicate experiments. ${ }^{* \star} P<0.01$. (d) Schematic model for HBV-stimulated Nrf2 activation and G6PD expression. In HBV-infected hepatocytes, HBx on the one hand results in the accumulation of p62 through inhibition of autophagic flux, and on the other hand interacts with Keap1 through p62, enabling the formation of HBx-p62-Keap1 aggregates in the cytoplasm. The aggregates hijack Keap1 from Nrf2 leading to Nrf2 activation and G6PD expression

bring new insights into the field. Viruses rely primarily on the metabolic capabilities of host cell to provide macromolecular precursors and energy to fuel their replication. ${ }^{25,26}$ It is known that Hepatitis $C$ virus and human cytomegalovirus are capable of enhancing nucleotide and lipid biosynthesis through upregulating glycolysis in their host cells. ${ }^{27,28}$ Enhancement of the PPP by HBx-mediated upregulation of G6PD may explain on one hand that metabolic perturbations such as the use of nucleoside analogs can suppress $\mathrm{HBV}$ production, ${ }^{29,30}$ and on the other hand the essential role of HBx in HBV replication. ${ }^{31}$ It is reasonable to speculate that through elevation of G6PD, HBV promotes PPP enabling host cell to utilize glucose for the biosynthesis of macromolecules to support their expensive proliferation which may be closely related to the development of HBV-associated HCC.

Notably, this is the first study showing G6PD overexpression in HBV-associated HCC, which correlates well with the results demonstrating HBx upregulates G6PD in cultured hepatocytes. As a pivotal initiator of the PPP, G6PD is highly regulated at the level of transcription, and in many cases by the transcription factor SREBP. ${ }^{32}$ Here, we have identified Nrf2 as a regulator of G6PD expression stimulated by HBx.
This conclusion is also supported by the study showing that Nrf2 activation redirects glucose into anabolic pathways by transcriptional activation of PPP genes including G6PD. ${ }^{15}$ Nevertheless, our data do not exclude a potential involvement of other transcriptional regulators such as hypoxia-inducible factor 1 (HIF-1) in HBx-stimulated G6PD expression, because it has recently been demonstrated that activation of HIF-1 can induce G6PD expression, ${ }^{33}$ and $\mathrm{HBx}$ is able to activate $\mathrm{HIF-1}{ }^{34}$

Considering that Nrf2 serves as an intracellular redox sensor and a master transcriptional regulator for a great many antioxidants, and G6PD-mediated PPP pathway is responsible for NADPH production, HBV-stimulated Nrf2 activation might also be a result of elevated intracellular ROS, because $\mathrm{HBx}$ is able to increase ROS levels. ${ }^{24,35}$ Nevertheless, we revealed that the activation of $\mathrm{Nrf} 2$ by $\mathrm{HBx}$ is mediated by an alternative more direct way. Clearly, we show that through interacting with the adaptor protein $\mathrm{p} 62$, $\mathrm{HBx}$ results in the formation of HBx-p62-Keap1 complexes, which further accumulates to generate the aggregates of the proteins in the cytoplasm. Identification of the interaction domain in p62 and application of the p62 mutant that failed to interact with 
Keap1 allowed us to determine that the formation of the complexes is initiated by $\mathrm{HBx}$, and $\mathrm{p} 62$ has a pivotal role in bridging $\mathrm{HBx}$ and Keap1. Further, real-time imaging and photobleaching techniques ensured the investigation of the mobility of these proteins in living cells. Our data indicate that the HBx-p62-Keap1 complexes immobilize Keap1 in the aggregates thereby prevent Nrf2 from the inhibition by Keap1, leading to the activation of Nrf2.

On the basis of our findings, and in coordination with the observation that HBV results in the accumulation of intracellular p62 by $\mathrm{HBx}$-mediated inhibition of autophagic flux, ${ }^{19}$ we propose a model for HBV-stimulated Nrf2 activation and G6PD expression (Figure 6). Our results suggest a potential impact of $\mathrm{HBx}$ on glucose metabolism of hepatocytes, which may be critical to the development of HBV-associated HCC.

\section{Materials and Methods}

Cell culture, transfection, and RNAi. HepG2, HepG2.2.15, and Huh7 cells were grown in DMEM with $10 \%$ FBS at $37^{\circ} \mathrm{C}$ under $5 \% \mathrm{CO}_{2}$. Transient transfection was performed using Lipofectamine 2000 (Invitrogen, Carlsbad, CA, USA) according to the manufacturer's instructions. Cell lines stably expressing HBXGFP were created by transient transfection followed by selection with $0.8 \mathrm{mg} / \mathrm{ml}$ G418 (Sigma-Aldrich, St. Louis, MO, USA).

All the siRNA duplexes were purchased from GenePharma (Shanghai, China). siRNA duplexes designed against conserved targeting sequences were transfected into cells at a final concentration of $20 \mathrm{nM}$ using Lipofectamine 2000 as specified by the manufacturer. The following siRNA duplexes were used: $5^{\prime}$-GCAUUGAA GUUGAUAUCGAU-3' for p62; 5'-AAGAGUAUGAGCUGGAAAAAC-3' for Nrf2; and $5^{\prime}$-AAGACCAAUUUCAGCAGACAGTT-3' for control siRNA. Expression plasmids for shRNAs were made in a pGLVH1/GFP+Puro vector. The targeted sequences were G6PD shRNA 5'-GTCGTCCTCTATGTGGAGAAT-3'; Nrf2 ShRNA 5'-CCGGCATT TCACTAAACACAAC-3'; control shRNA 5'-TTCTCCGAACGTGTCACGTT-3'. Stable shRNA transfectants were selected in medium containing $1 \mu \mathrm{g} / \mathrm{ml}$ puromycin (Sangon Biotech, Shanghai, China).

Antibodies, reagents, and plasmids. The following antibodies were used: anti- $\beta$-actin, anti-G6PD (Sigma-Aldrich); anti-HBcAg (Abcam, Cambridge, MA, USA), anti-Keap1 (Cell Signaling, Danvers, MA, USA); anti-Myc, anti-Nrf2 (sc365949), anti-Lamin B, anti-p62, and anti-Flag (Santa Cruz, Santa Cruz, CA, USA); anti-GFP (BD Biosciences, Franklin Lakes, NJ, USA); goat anti-rabbit IRDye800CW and goat anti-mouse IRDye680 (LI-COR Biosciences, Lincoln, NE, USA). Plasmids with the 1.3mer HBV genomic DNA (pUC19 HBV), the HBx-negative 1.3mer HBV genomic DNA (pUC19 HBVX $^{-}$) and HBx-GFP were described previously. ${ }^{19,36}$ Dsred-Keap1 was provided by Yue Xiong (University of North Carolina at Chapel Hill, Chapel Hill, NC, USA). For Flag-p62 construction, the p62 coding region was amplified from Cherry-GFP-p62, a gift from Terje Johansen (University of Tromsø, Tromsø, Norway), and was inserted into pCMV-Tag 2A vector using the EcoRI and Xhol restriction sites. HBx-Myc was made by cloning the CDNA of $\mathrm{HBx}$ into pcDNA-3.1 vector using EcoRl and Xhol restriction sites. The point mutation for Flag-p62 T350A and Dsred-Keap1 N382A was created by PCR-based site-directed mutagenesis using primer: 5'-AAGAAGTGGACCCGTCTGCAGGTGAACTCCAGT CC-3' (p62-sense), 5'-GGACTGGAGTTCACCTGCAGACGGGTCCACTTCTT-3' (p62-antisense), 5'-CGTGGGCGGCAGGGCCAACTCGCCCGAC-3' (Keap1-sense) and 5'-GTCGGGCGAGTTGGCCCTGCCGCCCACG-3' (Keap1-antisense).

Human tissues. Frozen fresh liver or liver cancer tissues were anonymously taken from the First Affiliated Hospital, Zhejiang University School of Medicine. Tumor liver tissues and their peripheral non-tumor tissues after surgical resection were collected from HCC patients with chronic HBV infection. Normal liver tissues after surgical resection were collected from patients with liver hemangiomas. The diagnoses were based on clinical laboratory examination. All the human tissues were taken with written informed consent and with the approval of the Medical Ethical Committee of Zhejiang University School of Medicine (No. 1-009). The study was conducted in accordance with the Helsinki Declaration of 1975, as revised in 1983.
Cell fractionation. About $3 \times 10^{6}$ cells were washed with PBS and resuspended in $200 \mu$ l of buffer A (10 mM HEPES pH 7.9, $10 \mathrm{mM} \mathrm{KCl}, 1.5 \mathrm{mM} \mathrm{MgCl}_{2}, 0.34 \mathrm{M}$ sucrose, $10 \%$ glycerol, $1 \mathrm{mM}$ dithiothreitol, $0.1 \%$ Triton $\mathrm{X}-100$ ) supplemented with a complete protease inhibitor cocktail (Roche, Indianapolis, IN, USA). The cells were incubated for $10 \mathrm{~min}$ on ice. Nuclei were collected in the pellet by low speed centrifugation $\left(1500 \times g, 5 \mathrm{~min}, 4^{\circ} \mathrm{C}\right)$. Nuclei were washed twice with buffer $\mathrm{A}$ without $0.1 \%$ Triton X-100, then lysed and denatured with sodium dodecyl sulfate (SDS) loading buffer. The Nrf2 levels in nuclei were analyzed by western blotting.

Western blot and immunoprecipitation. Western blot was performed as described previously. ${ }^{19}$ Briefly, proteins obtained from lysed cells were denatured and loaded on SDS polyacrylamide gel. Afterwards, the proteins were transferred onto PVDF membranes, blocked in TBS-T $(150 \mathrm{mmol} / \mathrm{L} \mathrm{NaCl}, 10 \mathrm{mmol} / \mathrm{L}$ Tris- $\mathrm{HCl} \mathrm{pH} 7.5$, and $0.1 \%$ Tween-20) containing $5 \%(\mathrm{w} / \mathrm{v})$ bovine serum albumin, and incubated with the corresponding primary and secondary antibodies. The specific bands were analyzed by the western blot infrared imaging system (LI-COR Biosciences). For immunoprecipitation, cells were lysed with nonidet P40 lysis buffer $(50 \mathrm{mM}$ Tris$\mathrm{HCl} \mathrm{pH} 7.5,100 \mathrm{mM} \mathrm{NaCl}, 1 \% \mathrm{NP}-40,1 \mathrm{mM}$ EDTA, $1 \mathrm{mM}$ DTT, 10\% glycerol) containing protease inhibitors. After centrifugation, the supernatants were incubated with antibody overnight and then Protein $A / G$ agarose for $2 \mathrm{~h}$ at $4{ }^{\circ} \mathrm{C}$. Immunocomplexes were washed and analyzed by western blotting.

G6PD enzyme activity. The enzyme activity of G6PD was assessed using a G6PD assay kit (Sigma, St. Louis, MO, USA) according to the manufacturer's instructions.

Immunostaining and confocal microscopy. For immunostaining, cells or frozen tissue sections were fixed in $4 \%$ formaldehyde. After washing twice with PBS, they were incubated in PBS/FBS (PBS, pH 7.4, containing 10\% FBS) to block non-specific sites of antibody adsorption. Then, the cells or sections were incubated with appropriate primary and secondary antibodies in $0.1 \%$ saponin as indicated in the figure legends. Images were captured on a Zeiss LSM510 Meta laser scanning confocal microscope (Carl Zeiss, Thornwood, NY, USA) with a 63 Plan Apochromat 1.4 NA objective.

For live-cell imaging, cells in chambers were imaged on a live cell station. Photobleaching was performed using an appropriate laser line at full power. For FRAP analysis, a selected region of a cell was photobleached and the fluorescence recovery of the region was monitored at low intensity illumination. For FLIP analysis, a selected region was repetitively photobleached and the loss of fluorescence from regions outside the photobleached region was monitored. For quantification of fluorescence intensity, non-saturated images were taken with a fully open pinhole, whereas non-quantitative images were obtained with a pinhole diameter equivalent to 1-2.5 Airy units.

Quantitative PCR. Total cellular RNA was isolated using TRIzol reagent (Invitrogen) and was reverse transcribed using M-MLV Reverse Transcriptase (Promega, Madison, WI, USA) according to the manufacturer's protocol. GAPDH was used as an invariant housekeeping gene internal control. Quantitative PCR was performed using primers as follows: (1) G6PD-sense, 5'-TGA CCTGGCCAAGAAGAAGA-3' and G6PD-antisense, 5'-CAAAGAAGTCCTCCAGCT TG-3'; (2) Nqo1-sense, 5'-GGAGAGTTTGCTTACACTTACGC-3' and Nqo1antisense, 5'-AGTGGTGATGGAAAGCACTGCCTTC-3'; (3) Gstm1-sense, 5'-TG CCCATGATACTGGGGTA-3' and Gstm1-antisense, 5'-GCCACTGGCTTCTG-TC ATAAT-3'; (4) Cyp2a5-sense, 5'-ACCAAGGACACCAAGTTTCG-3' and Cyp2a5antisense, 5'-AGAGCCCAGCATAGGAAACA-3'; (5) GAPDH-sense, 5'-GGAGCCA AAAGGGTCATCATCT-3' and GAPDH-antisense, 5'-GAGGAGCCATCCACAGTCTTCT-3'.

Cell proliferation and colony formation assay. HBx-GFP cell lines were transfected with shRNAs as indicated in the figure legends, and shRNAexpressing cells were selected in medium containing puromycin for 3 weeks. For cell proliferation assay, the cells were seeded in 24-well plates in triplicate at a density of 2000 cells per well in $0.5 \mathrm{ml}$ of medium. Cell number at the indicated time points was determined by counting using a haemocytometer. Colony formation assay in soft agar was performed as previously described. ${ }^{37}$ Briefly, $5 \times 10^{3}$ cells per well were resuspended in DMEM containing $0.3 \%$ agar. Then, the suspension was laid over DMEM containing $0.5 \%$ agar in each of the triplicate wells of a 6 -well plate. The plates were incubated for 14 days in a $5 \% \mathrm{CO}_{2}$ incubator at $37^{\circ} \mathrm{C}$, with replenishment of medium every 3 days. Colonies were imaged using Nikon ECLIPSE TI microscopy and colonies in three randomly chosen fields per well were counted for quantification. 
Statistical analysis. All data were presented as means \pm S.E.M. Statistical significance of the differences in the experimental data was determined using the Student's $t$-test. Differences were considered as significant at values of $P<0.05$.

\section{Conflict of Interest}

The authors declare no conflict of interest.

Acknowledgements. We thank Drs Jing-hsiung Ou, Yue Xiong, Terje Johansen and Zheng-gang Yang for sharing plasmids pUC19 HBV, pUC19 HBVX', Dsred-Keap1, Cherry-GFP-p62, and HBsAg-GFP, respectively. This work was supported by National Basic Research Program of China (2011CB910100 and 2013CB910200), National Natural Science Foundation of China (31530040, 31171288 and 31271431) and China Postdoctoral Science Foundation (2014M560478).

1. Vander HM, Cantley LC, Thompson CB. Understanding the Warburg effect: the metabolic requirements of cell proliferation. Science 2009; 324: 1029-1033.

2. Deberardinis RJ, Sayed N, Ditsworth D, Thompson CB. Brick by brick: metabolism and tumor cell growth. Curr Opin Genet Dev 2008; 18: 54-61.

3. Bokun R, Bakotin J, Milasinovic D. Semiquantitative cytochemical estimation of glucose-6phosphate dehydrogenase activity in benign diseases and carcinoma of the breast. Acta Cytol 1987; 31: 249-252.

4. Wang J, Yuan W, Chen Z, Wu S, Chen J, Ge J et al. Overexpression of G6PD is associated with poor clinical outcome in gastric cancer. Tumour Biol 2012; 33: 95-101.

5. Zampella EJ, Bradley EJ, Pretlow TN. Glucose-6-phosphate dehydrogenase: a possible clinical indicator for prostatic carcinoma. Cancer 1982; 49: 384-387.

6. Hu T, Zhang C, Tang Q, Su Y, Li B, Chen L et al. Variant g6pd levels promote tumor cell proliferation or apoptosis via the STAT3/5 pathway in the human melanoma xenograft mouse model. BMC Cancer 2013; 13: 251

7. Kuo W, Lin J, Tang TK. Human glucose-6-phosphate dehydrogenase (G6PD) gene transforms NIH 3T3 cells and induces tumors in nude mice. Int J Cancer 2000; 85: 857-864.

8. Jiang P, Du W, Wang X, Mancuso A, Gao X, Wu M et al. p53 regulates biosynthesis through direct inactivation of glucose-6-phosphate dehydrogenase. Nat Cell Biol 2011; 13: 310-316.

9. Du W, Jiang P, Mancuso A, Stonestrom A, Brewer MD, Minn AJ et al. TAp73 enhances the pentose phosphate pathway and supports cell proliferation. Nat Cell Biol 2013; 15 : $991-1000$.

10. Jaramillo MC, Zhang DD. The emerging role of the Nrf2-Keap1 signaling pathway in cancer. Genes Dev 2013; 27: 2179-2191.

11. Shibata T, Kokubu A, Gotoh M, Ojima H, Ohta T, Yamamoto M et al. Genetic alteration of Keap1 confers constitutive Nrf2 activation and resistance to chemotherapy in gallbladder cancer. Gastroenterology 2008; 135: 1358-1368.

12. Singh A, Misra V, Thimmulappa RK, Lee H, Ames S, Hoque MO et al. Dysfunctional KEAP1-NRF2 interaction in non-small-cell lung cancer. PLoS Med 2006; 3: e420.

13. Nioi $P$, Nguyen T. A mutation of Keap1 found in breast cancer impairs its ability to repress Nrf2 activity. Biochem Biophys Res Commun 2007; 362: 816-821.

14. Inami $Y$, Waguri S, Sakamoto A, Kouno T, Nakada K, Hino $\mathrm{O}$ et al. Persistent activation of Nrf2 through p62 in hepatocellular carcinoma cells. J Cell Biol 2011; 193: 275-284.

15. Mitsuishi $\mathrm{Y}$, Taguchi K, Kawatani $\mathrm{Y}$, Shibata T, Nukiwa T, Aburatani $\mathrm{H}$ et al. Nrf2 redirects glucose and glutamine into anabolic pathways in metabolic reprogramming. Cancer Cell 2012; 22: 66-79.

16. Bauer AK, Cho HY, Miller-Degraff L, Walker C, Helms K, Fostel $\mathrm{J}$ et al. Targeted deletion of Nrf2 reduces urethane-induced lung tumor development in mice. PLOS One 2011; 6: e26590.

17. DeNicola GM, Karreth FA, Humpton TJ, Gopinathan A, Wei C, Frese K et al. Oncogene-induced Nrf2 transcription promotes ROS detoxification and tumorigenesis. Nature 2011; 475: 106-109.

18. Ichimura Y, Waguri S, Sou YS, Kageyama S, Hasegawa J, Ishimura R et al. Phosphorylation of p62 activates the Keap1-Nrf2 pathway during selective autophagy. Mol Cell 2013; 51: 618-631.

19. Liu B, Fang M, Hu Y, Huang B, Li N, Chang C et al. Hepatitis B virus $X$ protein inhibits autophagic degradation by impairing lysosomal maturation. Autophagy 2014; 10: 416-430.
20. Komatsu M, Kurokawa H, Waguri S, Taguchi K, Kobayashi A, Ichimura Y et al. The selective autophagy substrate p62 activates the stress responsive transcription factor Nrf2 through inactivation of Keap1. Nat Cell Biol 2010; 12: 213-223.

21. Bjorkoy G, Lamark T, Brech A, Outzen H, Perander M, Overvatn A et al. p62/SQSTM1 forms protein aggregates degraded by autophagy and has a protective effect on huntingtin-induced cell death. J Cell Biol 2005; 171: 603-614.

22. Chung TW, Lee YC, Ko JH, Kim CH. Hepatitis B Virus $\mathrm{X}$ protein modulates the expression of PTEN by inhibiting the function of $p 53$, a transcriptional activator in liver cells. Cancer Res 2003; 63: 3453-3458.

23. Lian Z, Liu J, Pan J, Satiroglu Tufan NL, Zhu M et al. A cellular gene up-regulated by hepatitis $B$ virus-encoded $X$ antigen promotes hepatocellular growth and survival. Hepatology 2001 34: $146-157$.

24. $\mathrm{Ha} \mathrm{HL}, \mathrm{Yu}$ DY. HBx-induced reactive oxygen species activates hepatocellular carcinogenesis via dysregulation of PTEN/Akt pathway. World J Gastroenterol 2010; 16: 4932-4937.

25. Munger J, Bajad SU, Coller HA, Shenk T, Rabinowitz JD. Dynamics of the cellular metabolome during human cytomegalovirus infection. PLoS Pathog 2006; 2: e132.

26. Vastag L, Koyuncu E, Grady SL, Shenk TE, Rabinowitz JD. Divergent effects of human cytomegalovirus and herpes simplex virus-1 on cellular metabolism. PLOS Pathog 2011; 7: e1002124.

27. Diamond DL, Syder AJ, Jacobs JM, Sorensen CM, Walters KA, Proll SC et al. Temporal proteome and lipidome profiles reveal hepatitis $C$ virus-associated reprogramming of hepatocellular metabolism and bioenergetics. PLoS Pathog 2010; 6: e1000719.

28. Munger J, Bennett BD, Parikh A, Feng XJ, McArdle J, Rabitz HA et al. Systems-level metabolic flux profiling identifies fatty acid synthesis as a target for antiviral therapy. Nat Biotechnol 2008; 26: 1179-1186

29. Lai CL, Gane E, Liaw YF, Hsu CW, Thongsawat S, Wang Y et al. Telbivudine versus lamivudine in patients with chronic hepatitis B. N Engl J Med 2007; 357: 2576-2588.

30. Hadziyannis SJ, Tassopoulos NC, Heathcote EJ, Chang TT, Kitis G, Rizzetto M et al. Adefovir dipivoxil for the treatment of hepatitis $B$ e antigen-negative chronic hepatitis $B$ N Engl J Med 2003; 348: 800-807.

31. Zoulim F, Saputelli J, Seeger $C$. Woodchuck hepatitis virus $X$ protein is required for viral infection in vivo. J Virol 1994; 68: 2026-2030.

32. Duvel K, Yecies JL, Menon S, Raman P, Lipovsky Al, Souza AL et al. Activation of a metabolic gene regulatory network downstream of mTOR complex 1. Mol Cell 2010; 39: 171-183.

33. Gao L, Mejías R, Echevarría M, López-Barneo J. Induction of the glucose-6-phosphate dehydrogenase gene expression by chronic hypoxia in PC12 cells. FEBS Lett 2004; 569 256-260.

34. Yoo YG, Oh SH, Park ES, Cho H, Lee N, Park H et al. Hepatitis B virus X protein enhances transcriptional activity of hypoxia-inducible factor-1alpha through activation of mitogenactivated protein kinase pathway. J Biol Chem 2003; 278: 39076-39084.

35. Lim W, Kwon SH, Cho H, Kim S, Lee S, Ryu WS et al. HBx targeting to mitochondria and ROS generation are necessary but insufficient for HBV-induced cyclooxygenase-2 expression. J Mol Med 2010; 88: 359-369.

36. Sir D, Tian Y, Chen WL, Ann DK, Yen TS, Ou JH. The early autophagic pathway is activated by hepatitis B virus and required for viral DNA replication. Proc Natl Acad Sci USA 2010; 107 4383-4388.

37. Xu J, Yun $X$, Jiang J, Wei $Y$, Wu Y, Zhang $W$ et al. Hepatitis B virus $X$ protein blunts senescence-like growth arrest of human hepatocellular carcinoma by reducing Notch1 cleavage. Hepatology 2010; 52: 142-154.

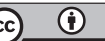

Cell Death and Disease is an open-access journal published by Nature Publishing Group. This work is licensed under a Creative Commons Attribution 4.0 International License. The images or other third party material in this article are included in the article's Creative Commons license, unless indicated otherwise in the credit line; if the material is not included under the Creative Commons license, users will need to obtain permission from the license holder to reproduce the material. To view a copy of this license, visit http://creativecommons.org/licenses/by/4.0/ 